\title{
Prensa local, menores y conflicto armado colombiano: análisis y efectos en la agenda pública
}

\author{
Giovanni Bohórquez-Pereira* \\ Ledis Bohórquez-Farfán ${ }^{\star *}$ \\ Beatriz Puyana-Mejía ${ }^{\star * *}$
}

* Candidato a Doctor en Ciencia Política. Profesor de la Facultad de Comunicación Social-Periodismo, Escuela de Ciencias Sociales, Universidad Pontificia Bolivariana, Bucaramanga, Colombia. Correo electrónico: giovanni.bohorquez@upb.edu.co

** Candidata a Doctor en Paz, Derechos Humanos y Desarrollo Sostenible. Profesora del departamento de Formación Humanística, Escuela de Ciencias Sociales, Universidad Pontificia Bolivariana, Bucaramanga, Colombia.

Correo electrónico: ledis.bohorquez@upb.edu.co

*** Abogada. Profesora, Universidad Cooperativa de Colombia, Bucaramanga, Colombia. Correo electrónico: beatriz.puyana@campusucc.edu.co

Recibido: 17 de septiembre del 2014 Aprobado: 28 de octubre del 2014

Cómo citar este artículo: Giovanni Bohórquez-Pereira, Ledis Bohórquez-Farfán y Beatriz Puyana-Mejía. Prensa local, menores y conflicto armado colombiano: análisis y efectos en la agenda pública. DIxI 20. Diciembre 2014. Pág. 45. doi: http://dx. doi.org/10.16925/di.v16i20.834

\section{Resumen}

El artículo presenta los resultados de la investigación "Caracterización de la información que circula en los periódicos locales Vanguardia Liberal y El Frente sobre niños y niñas víctimas del conflicto armado". Es un estudio exploratorio-descriptivo que analiza, desde la técnica análisis de contenido, las estructuras de cincuenta y seis notas periodísticas de ciento cuatro periódicos revisados y en su interior, la exposición del sujeto investigado. Tanto el proceso de investigación como el análisis fueron desarrollados a la luz de los fundamentos legales referentes a los derechos de los menores inmersos en el conflicto armando y a su protección integral, contenidos en la Constitución Política de Colombia y los convenios internacionales. Se encontró que en la noticia - el género de mayor registro (58\%) - es reducida la presencia del niño o niña víctima del conflicto como fuente informativa (6\%); y no hay registro de análisis del tema en columnas de opinión y editoriales. En síntesis, los niños y niñas se tienen en cuenta cuando hay situaciones que ameritan la publicación del hecho noticioso, con énfasis en lo judicial y son limitados los artículos que promueven una mirada desde la perspectiva del respeto y garantía de sus derechos.

Palabras clave: derechos de los niños, conflicto armado, opinión pública, protección legal.

Local Press, Minors and the Colombian Armed Conflict: Analysis and Effects on the Public Agenda

\section{Abstract}

This article presents the results of the research work "Characterization of Information Circulating in the Local Newspapers Vanguardia Liberal and El Frente about Boys and Girls who are Victims of the Armed Conflict." It is a descriptive and exploratory study that, using the content analysis technique, analyzes the structures of 56 articles in 104 newspapers and how they reported on the topic. Both the research process and the analysis were carried out in light of legal premises referring to the rights of minors immersed in an armed conflict and their comprehensive protection, as contained in the Political Constitution of Colombia and international conventions. It was found that in the news - which accounted for a major part of the publications (58\%) - there is very little coverage on boys and girls who are victims of the armed conflict as information sources (6\%); and there is no record of analysis of this topic in the editorials and opinion pieces. In summary, boys and girls are taken into account when there are situations that merit publication of a newsworthy event, with an emphasis on the judicial aspect, whereas articles that promote an approach from the perspective of respect and guarantees for their rights are limited.

Keywords: rights of children, armed conflict, public opinion, legal protection.

IMPRENSA LOCAL, MENORES E CONFLITO ARMADO COLOMBIANO: ANÁLISE E EFEITOS NA AGENDA PÚBLICA

\section{Resumo}

O artigo apresenta os resultados da investigação "Caracterização da informação publicada nos jornais locais Vanguardia Liberal e El Frente sobre crianças vítimas do conflito armado". Trata-se de um estudo exploratório-descritivo que analisa, desde a técnica análise de conteúdo, as estruturas de cinquenta e seis notas jornalísticas de cento e quatro jornais revisados e dentro, a exposição do sujeito pesquisado. Tanto o processo de investigação quanto a análise foram desenvolvidos seguindo os fundamentos legais referentes aos direitos dos menores imersos no conflito armando e à sua proteção integral, contidos na Constituição Política da Colômbia e os convênios internacionais. Encontrou-se que na notícia -o gênero de maior registro (58\%) - é reduzida a presença da criança vítima do conflito como fonte informativa (6\%); e não há registro de análise do assunto em colunas de opinião ou editoriais. Em resumo, crianças são levadas em conta quando existem situações que mereçam a publicação do fato noticioso, com ênfase no judiciário e são restritos os artigos que promovem um olhar desde a perspectiva do respeito e garantia de seus direitos.

Palavras-chave: direitos das crianças, conflito armado, opinião pública, proteção legal. 


\section{INTRODUCCIÓN}

El problema de los desplazamientos internos de la población civil que ha venido sufriendo Colombia desde hace más de cincuenta años, constituye uno de los factores más significativos de la crisis que afecta a todos los estamentos de la sociedad.

Este fenómeno actualmente involucra a todos los actores sociales: el Estado, la empresa privada, los políticos, la sociedad civil, la academia y la Iglesia. Pero cuando se trata de los menores, el problema asume connotaciones especiales en relación con sus derechos como víctimas del conflicto armado, ya que, a su vez, en ocasiones los menores se convierten en victimarios dentro de la guerra, situación que se erige como una de las expresiones más preocupantes en la degradación de la guerra civil.

Dentro de un país en permanente conflicto, la garantía de los derechos fundamentales es una misión compleja para el Estado, más aún cuando se trata de los niños y adolescentes. El 20 de noviembre de 1989, la Organización de Naciones Unidas logra consolidar la Convención sobre los Derechos del Niño (CDN), la cual permite crear un marco legal de protección para los menores que hasta ese momento se debatían en medio de las confrontaciones armadas sin que el Estado ofreciera garantías: "Más que un catálogo de derechos, la CDN es una declaración completa de las obligaciones que los Estados y las sociedades deben cumplir con los niños, niñas y adolescentes".

La Ley 12 de 1991, por medio de la cual el Congreso de la República aprobó la Convención Internacional sobre los Derechos del Niño, de noviembre de 1989, en su artículo 39 establece:

Artículo 39: Los Estados Partes adoptarán todas las medidas apropiadas para promover la recuperación física y psicológica y la reintegración social de todo niño víctima de: cualquier forma de abandono, explotación o abuso; tortura u otra forma de tratos crueles, inhumanos o degradantes; o conflictos armados. Esta recuperación y reintegración se llevará a cabo en un ambiente que fomente la salud, el respeto de sí mismo y la dignidad del niño.
Estos primeros esfuerzos por proteger los derechos de la infancia y la adolescencia integran protocolos facultativos que permiten evidenciar problemáticas constantes que afectan a la niñez, legislando alrededor de temas tales como la venta de niños, la prostitución infantil y la utilización de niños en la pornografía, además de lo relativo a la participación de niños en los conflictos armados. Entraron en vigencia desde el 2002, año en que se evidencia un despertar en la temática de los menores dentro del conflicto armado.

En la década de los noventa, gracias a las consultas realizadas por Unicef y otras organizaciones internacionales, el mundo comenzó a prestar especial atención al impacto causado por la guerra en los niños, con lo que se generó un cambio en la forma de apreciarlos. Son considerados como seres capaces de contemplar el mundo sin que por ello se conviertan en adultos pequeños. En Colombia, el Código de la Infancia y la Adolescencia (CIA), en su artículo $3 .^{\circ}$, estipula:

Sujetos titulares de derechos. Para todos los efectos de esta ley son sujetos titulares de derechos todas las personas menores de 18 años. Sin perjuicio de lo establecido en el artículo 34 del Código Civil, se entiende por niño o niña las personas entre los 0 y los 12 años, y por adolescente las personas entre 12 y 18 años de edad. ${ }^{2}$

Esta ley constituyó un importante aporte para la protección de los derechos de los niños y adolescentes, así como para la garantía de su pleno y armonioso desarrollo, de manera que tengan la posibilidad de crecer en el seno de la familia y en un ambiente sano. Desarrolló el precepto constitucional consagrado en los arts. 44 y 45 de la Carta Magna, en relación con la protección de los menores, sus libertades y las obligaciones de todos los estamentos públicos y privados que tengan a cargo su protección, educación y progreso.

La Resolución 1612 de 2005 del Consejo de Seguridad de Naciones Unidas contribuyó a establecer un marco general para la protección de los menores afectados por el conflicto armado. Se estableció un mecanismo para reunir y proporcionar información oportuna, objetiva, precisa y fiable acerca del reclutamiento y la utilización de niños en la guerra, proceso de información que Colombia voluntariamente acogió en el 2008.

\footnotetext{
2. Ley 1098 de 2006. Por la cual se expide el Código de la Infancia y la Adolescencia. Noviembre 8 de 2006. Do N. ${ }^{\circ} 46446$. Disponible en http:// www.alcaldiabogota.gov.co/sisjur/normas/Norma1.jsp?i=22106
}

1. United Nations International Children's Emergency Fund. LA Convención sobre los Derechos del Niño (CDN). (2013). Disponible en http://www.unicef.org/lac/CDN.pdf. Pág. 1. [De aquí en adelante UNICEF] 
El resultado perseguido por este procedimiento es el de conminar a los Estados a tomar las medidas necesarias para la protección integral de los derechos de los menores involucrados en el conflicto interno y, a su vez, a establecer una base de información sobre la vulneración de sus derechos.

Las principales dificultades que tiene el conflicto en los niños, niñas y adolescentes (NNA), definidas por la Resolución 1612 del Consejo de Seguridad de las Naciones Unidas, son: a) asesinato y mutilación, b) reclutamiento, c) violencia sexual, d) ataques a escuelas y hospitales, e) denegación de acceso a la asistencia humanitaria, f) secuestros y g) desplazamiento forzado en particular para el caso colombiano. ${ }^{3}$

A partir de la regulación internacional y nacional más específica sobre menores afectados por las guerras internas, se han emprendido investigaciones al respecto centradas en caracterizar (sustentando con cifras) aspectos tales como factores y motivaciones que favorecen la vinculación a los grupos armados, características de los jóvenes vinculados, así como la dinámica y las tareas específicas que desempeñan niños y niñas (cocina, mensajería, suministros y compañeros sexuales, entre otras).

Los datos globales refieren que un $50 \%$ de los niños y jóvenes que se integran al conflicto armado lo hacen por voluntad propia.

De ellos, al 33\% los atrajo las armas y los uniformes, a otro $33 \%$ fueron las condiciones de pobreza las que los llevaron a tomar esta decisión, al $16,60 \%$ porque crecieron conviviendo con ella, y el 8,33\% se vinculó por enamoramiento, decepción amorosa o sentimiento de venganza porque sus familias y bienes habían sido destruidos. ${ }^{4}$

En Santander - región colombiana donde se origina la presente investigación-, las edades de ingreso de los militantes a los grupos ilegales se encuentran concentradas en un 35\% en el rango de los 8 a 17 años de edad (es el porcentaje más alto del total). Entre las causas de vinculación se destaca la falta de empleo como la mayor motivación (33\%), junto con la curiosidad

\footnotetext{
3. Coalición contra la vinculación de niños, niñas y jóvenes al conflicto armado en Colombia. Boletin estadístico 2009-2011. Boletín DE MONItoreo NiñeZ y Conflicto Armado 9. Noviembre de 2012. Disponible en: http://www.coalico.org/Bonca_coalico_9.pdf [De aquí en adelante COALICO]

4. Julián Aguirre y Miguel Álvarez-Correa, Eds. Guerreros SIN SOMBRA: NIÑOS, NIÑAS Y JÓVENES VINCULADOS AL CONFLICTO ARMADO. Instituto Colombiano de Bienestar Familiar y Procuraduría General de la Nación. (2002). Pág. 32.
}

(16\%), el deseo de venganza (15\%), y en porcentajes menores la influencia del sitio o las amistades, el abandono o conflictos familiares, y sólo como obligación en $7 \%{ }^{5}$

Dentro de la Ley 1098, se encuentran concentrados diversos artículos que protegen al menor de las consecuencias del conflicto armado. El artículo 20 incluye como derechos de protección: 1) el abandono físico, emocional y psicoafectivo; 2) la explotación económica; 3) el consumo de tabaco, sustancias psicoactivas, estupefacientes o alcohólicas y la utilización, el reclutamiento o la oferta de menores en actividades de promoción, producción, recolección, tráfico, distribución y comercialización; 4) la violación, prostitución y la explotación sexual; 5) el secuestro, la venta, la trata de personas; 6) las guerras y los conflictos armados internos; 7) el reclutamiento y la utilización de los niños por parte de los grupos armados organizados al margen de la ley; 8) la tortura y toda clase de tratos y penas crueles, inhumanas, humillantes y degradantes, la desaparición forzada y la detención arbitraria; 9) la situación de vida en calle de los niños y las niñas; 10) los traslados ilícitos y su retención en el extranjero para cualquier fin; 11) el desplazamiento forzado; 12) el trabajo; 13) las peores formas de trabajo infantil; 14) el contagio de enfermedades infecciosas prevenibles; 15) los riesgos y efectos producidos por desastres naturales y demás situaciones de emergencia; 16) cuando su patrimonio se encuentre amenazado por quienes lo administren; 17) las minas antipersonales; 18) la transmisión del VIH-SIDA y las infecciones de transmisión sexual; 19) cualquier otro acto que amenace o vulnere sus derechos. ${ }^{6}$

En el marco del conflicto, los niños y adolescentes vinculados en su mayoría de manera voluntaria, son reconocidos por los medios y la sociedad como víctimas o victimarios, estando siempre en medio de dos papeles totalmente contradictorios. Puesto que

la vida cotidiana implica, para estos niños, niñas y jóvenes, estar propensos a los abusos y vejámenes más agresivos y violentos por parte de sus compañeros y superiores, pero también, los insta a ejercer su dominio frente a los enemigos o los débiles, mediante el poder de las armas y el uso de las tecnologías de la muerte.

\footnotetext{
5. Corporación para el Desarrollo del Oriente. Desarme, desmovilización y reintegración en Santander. Boletín 1. 2012. Disponible en http://www.corporacioncompromiso.org/index.shtml?apc=e1-1---\&x=599

6. Ley 1098 de 2006, supra, nota 2. Pág. 5.

7. Juan Carlos Amador-Baquiro. El intersticio de la víctima-victimario: un análisis de los procesos de subjetivación de cuatro desvinculados de grupos armados en Colombia. Universitas humanística 69. Junio de 2010.
} 
Finalmente, es importante reconocer la realidad en la que se desarrolla la vinculación, la motivación, la vida e incluso la desvinculación de los niños a los grupos armados, con el fin de poder narrar desde allí la guerra de los niños en Colombia. Es considerable el número de menores combatientes en las filas de los diferentes contendientes armados. Se calcula que cerca de 2000 son los menores que están en las filas de la guerrilla, y 3000 los que se encuentran vinculados con grupos paramilitares.

Es innegable la afectación del conflicto armado en los niños, niñas y adolescentes de manera directa o indirecta. Se les afecta porque se condiciona el contexto en el que nacen, crecen y se desarrollan, el cual tiende a convertirlos en víctimas y, no en pocas ocasiones, también en victimarios.

Las evidencias de los efectos del conflicto en NNA han sido registradas por la prensa nacional, regional y local, y particularmente por organizaciones no gubernamentales que han permitido a la sociedad colombiana conocer la situación y, en ocasiones, exigir la intervención estatal en su resolución.

El Observatorio Niñez y Conflicto Armado de la Coalición contra la vinculación de niños, niñas y jóvenes al conflicto armado en Colombia (COALICo) registró, para el período 2009-2011, 2269 violaciones e infracciones consumadas contra niños, niñas y adolescentes: "De manera preocupante, los homicidios, la desaparición forzada y el reclutamiento y uso de menores fueron los delitos que persistieron entre dichos años".

En los tres años de seguimiento de coAlico 2009-2011, se registraron 1733 delitos en 10 departamentos del país; 55 amenazas y 59 hechos de riesgo para los menores. Se presentaron hechos de uso y reclutamiento forzado y, sumado a todo ello, las consecuencias del desplazamiento forzado.

En el 2010, el observatorio registró cómo, en 26 de los 32 departamentos, se cometieron 450 violaciones contra niños, niñas y jóvenes. Los departamentos con mayor número de hechos fueron: Antioquia (45,8\% de los delitos); Meta (9,1\%); Arauca (8,7\%); y Putumayo $(8,7 \%)$. Los homicidios, las lesiones, el reclutamiento y uso de NNA, el desplazamiento forzado y el ataque a bienes civiles fueron las violaciones que se cometieron en mayor proporción.

8. COAlico, supra, nota 3. Pág. 4.
Finalmente, en el monitoreo del año 2011 se encontró que los homicidios, las desapariciones forzadas, el desplazamiento forzado y el reclutamiento y uso de NNA se presentaron con particular intensidad. En total, se registraron 86 violaciones en 10 departamentos (sumadas a 20 situaciones de amenaza), ocurridas especialmente en Antioquia (27,9\% de los delitos), Cundinamarca $(11,6 \%)$ y Valle del Cauca $(22,1 \%)$.

\section{A. Importancia de los medios y sus efectos en la opinión pública}

De acuerdo con la particularidad de la investigación "Caracterización de la información que circula en los periódicos Vanguardia Liberal y El Frente sobre niños y niñas víctimas el conflicto armado", la mirada sobre las afectaciones del conflicto en los niños y niñas, también involucra a la prensa y examina si la variedad de formas y la cantidad de víctimas infantiles del conflicto puede llegar incluso a aumentar, dependiendo de la manera como se informa sobre situaciones que los afectan e involucran.

Se ha intentado hacer una mirada y revisión de cómo estos medios registran la información relacionada con niños y niñas víctimas, con el objetivo de concientizar a los periodistas y a los empresarios de los medios y comprometerlos con la dignificación de los niños y niñas desde el manejo que se hace en el cubrimiento noticioso.

El manejo que los medios de comunicación dan a las noticias que involucran niños y niñas puede contribuir a movilizar a una sociedad en apoyo y solidaridad hacia ellos o, por el contrario, a rechazarlos. Quizás así la prensa contribuye a revictimizarlos. En tal sentido, se refuerza la influencia de la prensa en la posibilidad de construir una sociedad respetuosa de los derechos de sus niños.

La fuerza del lenguaje en la presentación de los hechos, la manera como se narra, las imágenes que se presentan de las situaciones, los verbos y los adjetivos que se utilizan puede intensificar la versión hacia una situación o persona; no en vano se ha dicho que los medios pueden encumbrar o tumbar un presidente, dado que la opinión pública está siendo manejada por ellos. Un ejemplo claro de la afirmación anterior son las conversaciones cotidianas entre amigos, en familia (incluso en la mesa), normalmente giran alrededor de las noticias del día, sin hacer mucho análisis, sencillamente repitiendo lo que el medio dice. 
Por esta razón, los periodistas tienen en sus manos una gran responsabilidad en el manejo de la información, como dice Correa, apoyada en Albert Chillón Asensio: ${ }^{9}$

Los silencios, las exclamaciones y los interrogantes, además de sustantivos, verbos y adjetivos que forman parte de ese recurso expresivo de los humanos, favorecen la representación del pensamiento pero dejan ver que "siempre existe una zona de claroscuro inevitable ente las palabras y su sentido".

Cuando el recurso del lenguaje se utiliza para informar sobre cualquier situación que es noticia, quien relata el hecho como periodista no sólo debe conocer la historia y encontrar las diversas versiones de verdad sobre ella, sino además tener el convencimiento de que las palabras desempeñan un papel crucial y no meramente instrumental, si desea mantener la línea de un periodismo crítico, cívico y éticamente responsable. ${ }^{10}$

La fuerza y sutileza de los mass media es tal que no siempre la cantidad de noticias significa mayor calidad en cuanto a la información que circula, así como tampoco permite una adecuada comprensión de la noticia en contexto. Menos aún, un seguimiento a las acciones que se presentan, cuando muchas veces no se acude a diversas fuentes que puedan mostrar las varias visiones y datos sobre el ítem que se esté trabajando.

De esta forma, los medios han contribuido a exacerbar las situaciones ya complejas, especialmente cuando anuncian cambios en situaciones del conflicto que no se han producido realmente o rompen con parámetros periodísticos que recomiendan no calificar ningún hecho para no delatar una carga de opinión parcial, como lo indica Carl N. Warren. ${ }^{11}$

En ese orden de ideas, Mauro Wolf, en 1987, enfatiza en la función especial del emisor. Asegura que en él reposa la responsabilidad de selección, construcción o elaboración del mensaje, "su difusión y la exclusión de algunas partes o, en ocasiones, de todo el mensaje", y señala que desde su perspectiva esta actitud se sigue presentando, por tanto la responsabilidad de lo que se presenta o publica en los medios tiene nombre propio,

9. Albert Chillón. El giro lingüístico en periodismo y su incidencia en la comunicación periodística. CuAdernos DE LA INFORMACIón 14. 2001. Pág. 35.

10. Mary Correa. El lenguaje de los medios que intensifica el conflicto armado colombiano. Reflexión Política 19. Junio de 2008. Pág. 107.

11. Id., pág. 108. y estará en sus manos el equilibrio informativo, la proyección y análisis del hecho registrado". ${ }^{12}$

En el libro Las trampas de la guerra, la organización Medios para la Paz señala cómo

el periodista a veces sigue el curso de automatismos condicionados y de mecanismos reflejos y a esto se le compara con el piloto automático que sigue conduciendo un avión, de acuerdo con una programación que previamente han hecho una serie de fuerzas, entre ellas, su bagaje como profesional de la información..$^{13}$

La manera como se presenta la información permite legitimar o deslegitimar un discurso, un hecho o una decisión. Basta con mirar de qué manera en el país el tema de la defensa de los derechos de los niños y niñas ha tomado fuerza y ha generado la reacción social cuando los medios lo convocan a través de sus discursos.

Por lo dicho anteriormente, es importante resaltar que así como los medios tienen el poder para arrasar y afectar, pueden hacerlo en diversas líneas de tal suerte que la afectación sea positiva. Por esta razón, al preguntarnos cuál puede ser el rol que juegan los medios de comunicación en la defensa de los derechos de la niñez, hacemos eco del planteamiento de Misle, el cual asevera:

Los medios de comunicación social son un pilar fundamental para las denuncias, cuando por acción u omisión, se violan o vulneran los derechos de la niñez y adolescencia. También son importantes para anunciar los avances y respuestas que desde los distintos ámbitos se están dando para hacer efectivos los derechos de la niñez en lo cotidiano ${ }^{14}$

Podemos identificar en la actualidad un avance en términos de la defensa y garantía de los derechos de los niños y niñas, en el que juega un papel importante el imaginario colectivo proclive a ello, y si bien es cierto que han confluido diversos intereses en este propósito, también lo es que los medios de comunicación han desarrollado un papel importante en la sensibilización de la sociedad colombiana hacia la defensa y garantía de los derechos de los niños y niñas.

12. Mauro Wolf. LA investigaCión DE LA COMUNiCACión DE MASAS. Crítica y Perspectivas. Editorial Paidós. (1987). Pág. 201.

13. Medios para la Paz. LAS trampas de LA GUerra: Periodismo y Conflicto. Corporación Medios para la Paz. (2001). Pág. 48.

14. Óscar Misle. LA NIÑEZ y LA AdOLESCENCIA EN LOS MEDIOS: ¿CUÁNDO sí y CUÁndo No? Ediciones El Papagayo. (2008). Pág. 23. 
De acuerdo con la Fundación Agencia de Comunicaciones Periodismo Aliado de la Niñez, el Desarrollo Social y la Investigación (PANDI), se ha presentado un cambio significativo en el manejo que los medios hacen en el tratamiento de las noticias relacionadas con la niñez, el cual se refleja en las denuncias hechas:

Muchas veces nos preguntamos ¿de qué le sirve a una niña de nueve años hablar cuando es agredida sexualmente y contarlo ante el juez si la Corte Suprema dice que ese "manoseo" solo es injuria? ¿De qué le sirve a un bebé llorar de dolor si es su padre el que lo está matando? En estos dos casos les sirvió porque los medios de comunicación hicieron que su voz fuera escuchada por los políticos, por los jueces y por la sociedad. Los fallos cambiaron y los agresores purgan penas por sus delitos. ${ }^{15}$

Por otra parte, se suma a lo expuesto hasta el momento un nuevo reto. Hay un gran desconocimiento de los mismos niños y niñas sobre sus derechos. José Alfredo Betancur, en el artículo "Convención sobre los Derechos del Niño 20 años de aciertos y desafíos" en el Informe anual de la PANDi 2008, recuerda que

en septiembre de 2005, la Coalición contra la Vinculación de Niños, Niñas y Jóvenes al Conflicto Armado presentó al Comité de los Derechos del Niño, órgano encargado de la vigilancia de la Convención, los resultados de una encuesta aplicada a 228 niños y niñas entre los 6 y 18 años, en distintas zonas del país. Según los resultados de ese estudio, solamente el $20 \%$ de ellos sabía qué es un derecho y conocía la Convención. De los entrevistados, el 14,2\% conoció sus derechos a través de organizaciones sociales, el $13,3 \%$ de su familia y solo el $31,5 \%$ de los maestros de sus escuelas sabía de su existencia. ${ }^{16}$

Ahora bien, hemos advertido la fuerza del lenguaje y en particular del lenguaje periodístico. Es desde allí que es posible retomar posturas teóricas, ahora desde la Opinión Pública (op). Un primer planteamiento se encuentra relacionado con el "clima de opinión",

15. Ximena Norato y Alexandra Farfán, Eds. LA HUella DE LA NiÑEZ eN la PRensa Colombiana 2010. Agencia de Comunicaciones Periodismo Aliado de la Niñez, el Desarrollo Social y la Investigación (PANDI), Personería de Medellín y Unicef. (2010).

16. Ximena Norato y Alexandra Farfán, Eds. LA HUElLA DE LA NiÑEZ eN la prensa Colombiana 2008. Agencia de Comunicaciones Periodismo Aliado de la Niñez, el Desarrollo Social y la Investigación (PANDI), Fundación Nuevo Periodismo Iberoamericano, Plan y Unicef. (2008). Pág. 14. concepto nacido de la corriente racionalista en el siglo XVIII y cuyo propósito, en su momento, fue defender las leyes racionales y su validez universal. Raúl Rivadeneira Prada recuerda cómo Carl Becker, en principio, utilizó este término para referirse a la importancia que en ese tiempo tenía la formación de opinión política y, en particular, lo relacionado con las ideas de los pensadores y precursores de la Revolución francesa.

Años después, y como resultado de las discusiones promovidas por los historiadores de la tradición clásica, se va indicar que el concepto se evidencia en la medida en que los hechos sociales que generan opinión trasciendan y perduren. Sin embargo, Paul F. Lazarsfeld indicará que, desde la Escuela empírica de la op, todo hecho influye y modifica cambios de mentalidad en los grupos sociales; por tanto, la opinión pública reacciona o responde ante situaciones que se le presenten o expongan:

En este sentido, lo importante no es sólo que ocurran, sino también la forman en que interpretan [...] la interpretación de los hechos no es necesariamente racional. En nuestras interpretaciones de un hecho particular pueden entrar muchos rasgos irracionales; a su vez, estos últimos afectarán nuestras opiniones y acciones respecto a aquél. ${ }^{17}$

Es decir, el "clima de opinión" es un complejo mecanismo presente en la convivencia social, que surge a partir de la emisión de mensajes (escritos, visuales y auditivos), los cuales logran, por su intensidad y los factores que los generan, cambiar pensamientos y actitudes, en principio de carácter individual y luego de forma colectiva.

Desde lo anterior, el registro que hacen de manera continua - por no decir diaria - los mass media sobre el conflicto armado colombiano, lleva a suponer que en este país se presentan los parámetros requeridos en el "clima de opinión". Las ediciones impresas (periódicos, revistas), y las emisiones radiales y audiovisuales (en particular noticieros), exponen a la sociedad los hechos relacionados con la guerra y sus diferentes actores (víctimas y victimarios). Esa reiteración lleva — según lo planteado- a que individual y colectivamente se tenga una posición sobre lo que ocurre.

Sin embargo, la forma en que presentan los periódicos título, extensión y ubicación del texto en la

17. Raúl Rivadeneira. La OPINIÓN PÚBLICA. ANÁLISIS, ESTRUCTURA Y MÉTOdos Para SU eStudio. Editorial Trillas. (2007). Pág. 118. 
página, el lenguaje periodístico utilizado a través de los géneros y la exposición visual (fotografías) del sujeto, sea este subversivo, militar, mujer, niño o niña, lleva a que los lectores interioricen el hecho social y expresen públicamente su reacción de consentimiento o rechazo.

No obstante, esa aprobación o negación del fenómeno social por parte de los individuos puede tener un condicionamiento adicional, el cual también es proporcionado por los medios masivos, denominada agenda-setting.

Este segundo aporte de la op fue reseñado en un principio por Walter Lippmann ${ }^{18}$ en 1922 cuando dijo, en su obra La opinión pública, que los editores y directores publican noticias sobre todos los temas, no para complacerlos, sino porque necesitan contactar a esa supuesta multitud de lectores interesada por la verdad. Décadas después, MacCombs y Shaw (1972) la formulan y ponen en evidencia la influencia que los medios ejercen sobre la opinión pública. ${ }^{19}$

Tanto D’Adamo, García y Freidenberg ${ }^{20}$ como Ló$\mathrm{pez}^{21}$ coinciden en que el estudio empírico publicado a principio de los setenta determinó los fundamentos de dicho postulado. McCombs y Shaw observaron una "correlación entre los temas de interés público y los temas de interés mediático, a través, nuevamente, de un estudio empírico parcial, en el que se pudo comprobar que el público conformaba su horizonte de expectativas de voto a partir de la información consumida en los medios".22

El mismo McCombs —dice López- afirmará que la mayoría de los conocimientos de las personas sobre lo público, o los temas que les llaman la atención y les generan preocupaciones no son propios de las personas, sino que se dan por lo entregado o difundido a través de los medios de comunicación, quienes actúan como la primera fuente de información. Esto lleva a que la sociedad centre su atención en algunos problemas y asuma un posición contraria con otros. "Ese modo de actuar de los medios modifica la aproximación de la

18. Véase Walter Lippmann. LA OpINIÓN PÚBLICA. Editorial C. de Langre. (2003).

19. Maxwell McCombs y Donald Shaw. The Agenda-Setting Function of Mass Media. Public Opinion Quarterly 2. Verano de 1972. Pág. 176.

20. Véase Orlando D’Adamo, Virginia García y Flavia Freidenberg. MEDios DE COMUNICACIÓN Y OPINIÓN PÚBLICA. McGraw-Hill. (2007).

21. Véase Guillermo López. COMUNICACión ELECTORAL Y FORMACión DE OPINIÓN PÚBLICA: LAS ELECCIONES GENERALES DE 2000 EN LA PRENSA ESPAÑOLA. Universidad de Valencia. (2004).

22. Id., pág. 167. gente a la realidad social, porque al describirla y precisarla instalan en el listado de los temas acerca de los cuales el público 'debe' discutir". 23

Por tanto, y siguiendo la línea teórica asumida, los medios masivos de información presentan a diario, en su diversas plataformas, la existencia de eventos y problemas. En otras palabras, visibilizan e invisibilizan acontecimientos, lugares y personas. Determinan en torno a qué temas debe orientarse la atención de los receptores, les definen prioridades y les establecen la interpretación que se debe hacer de lo ocurrido o registrado. "Así, la función de establecimiento de la agenda parece constituir el mapa que sirve de guía a la opinión pública en su travesía por los espacios públicos.".

Sobre estas bases teóricas y conceptuales se concentró el estudio que formuló como pregunta: ¿cuáles son las características de la información publicada sobre las niñas y niños víctimas del conflicto armado en los periódicos locales Vanguardia Liberal, El Frente de Bucaramanga y Santander? Así, trazó como objetivos: caracterizar el manejo que dan los medios impresos Vanguardia Liberal y El Frente a la información noticiosa que tiene directa o indirecta relación con los niños y niñas víctimas del conflicto; determinar los tipos de hechos que están exponiendo estos medios impresos sobre niños y niñas víctimas del conflicto; determinar el lugar (secciones del periódico) donde se registra mayor cubrimiento por parte de los dos medios sobre información relacionada con los menores víctimas del conflicto; y mostrar las características en cuanto al manejo de la información noticiosa en los dos medios impresos locales.

Cabe destacar, además de lo expuesto por la Constitucional sobre la responsabilidad de los medios y su obligación de proteger a los NNA en sus informes, que al respecto la Corte, en Sentencia T-453/13, expone como consideraciones fundamentales en la relación de los medios de comunicación con los NNA:

a) la responsabilidad social de los medios de comunicación y la obligación de protección y reserva frente a niños, niñas y adolescentes; b) el tratamiento de la infancia en los medios debe respetar el principio de universalidad, priorizar el interés superior del niño en la toma de decisiones, garantizar la exactitud y contextualización adecuada de los contenidos de las noticias sobre infancia y proteger la imagen e identidad de los

23. D’Adamo, García y Freidenberg, supra, nota 20. Pág. 122.

24. Id., Pág. 123. 
niños y niñas; c) comprobar de forma especialmente cuidadosa la fiabilidad de las fuentes; d) garantizar el derecho a la participación de los niños y niñas en los medios de comunicación; e) promover el rol de los medios de comunicación como agentes de sensibilización social; y f) garantizar la protección de los niños y niñas en los medios de comunicación. ${ }^{25}$

\section{MÉTODO}

La investigación se ubicó en el campo de la exploración-descripción, al considerarse como punto de partida para trabajos que relacionan el conflicto armado, los niños y la prensa escrita en el ámbito local. A este tipo de investigaciones, según Hernández Sampieri, Fernandez Collado y Baptista, ${ }^{26}$ anteceden trabajos de mayor alcance (correlacionales o explicativos), y además su carácter descriptivo permitió especificar propiedades y características del objeto de estudio.

El enfoque de la investigación se enmarca en el campo de lo cualitativo y en particular desde la hermenéutica, entendida como "la teoría de las reglas que gobiernan una exégesis, es decir, una interpretación de un texto particular o colección de signos susceptible de ser considerada como un texto,, ${ }^{27}$ dado que a través de la nota periodística se revisó lo ocurrido con los niños y niñas involucrados en el conflicto armado, para desde allí interpretar el fenómeno.

A partir de dicha decisión se acude a la técnica del análisis de contenido (AC), desde la cual se pueden realizar lecturas de la realidad e interpretaciones de los fenómenos sociales.

El AC centra su tarea en el orden sistemático, descriptivo, objetivo y contable del contenido presente y latente de la información, entendida esta como "un conjunto transracional que presenta reglas sintácticas, semánticas y pragmáticas, reglas de coherencia y cohesión, repercutidas en una práctica social en particular".28

25. Corte Constitucional de Colombia. Sentencia T-453/13. (M. P. Nilson Pinilla Pinilla; julio 15 de 2013). Disponible en http://www. corteconstitucional.gov.co/relatoria/2013/T-453-13.htm

26. Roberto Hernández Sampieri, Carlos Fernández y Pilar Baptista. MeTODOLOGía DE LA INVESTigación. McGraw Hill. (2010).

27. Carlos Sandoval. Investigación cualitativa. Programa de EsPeCIALIZación en Teoría, MÉtodos y TÉcnicas de Investigación Social. Arfo Editores. (2002). Pág. 67.

28. Klaus Krippendorff. Metodología de anÁlisis de Contenido, TEORÍA Y PRÁCTICA. (1997). Pág. 121

\section{A. Procedimiento}

Tomando como referente a Klaus Krippendorff, ${ }^{29}$ el estudio se enmarcó en el análisis semántico, el cual a su vez desarrolla en su procedimiento el análisis de designaciones, el análisis de atribuciones y el análisis de las aseveraciones. El análisis de designaciones posibilita establecer las frecuencias con las que se hace referencia a determinado objeto-sujeto; el análisis de atribuciones ofrece frecuencias a ciertas características de un objeto-sujeto de estudio, y el análisis de las aseveraciones otorga las frecuencias con que ciertos objetos-sujetos son caracterizados de un modo particular.

Lo anterior indica que al estudiar la información registrada por los periódicos locales de Bucaramanga, Vanguardia Liberal y El Frente, durante el año 2011, a partir del análisis de contenido de tipo semántico, se acordó entender como sujetos de estudio a los niños y niñas víctimas del conflicto. De igual forma, a partir de los reportes hechos por los medios impresos seleccionados y de su posterior estudio, se ubicaron y cuantificaron las frecuencias con que se hizo referencia a ellos, sus características generales y la condición de víctima o victimario.

Como unidad de muestreo, la investigación revisó la información periodística que publicaron los diarios Vanguardia Liberal y El Frente, en la cual se hizo referencia a los niños y niñas víctimas del conflicto armado.

Identificada la muestra de estudio y ubicada la información relacionada con lo definido en los objetivos específicos, se procedió a sistematizar la información. Se retomó y adaptó el procedimiento de BohórquezPereira $^{30}$ a través de rejillas de análisis definidas a partir de las categorías de jerarquización, lugar de registro informativo, sujeto y participación en la información.

\section{B. Muestra}

La investigación utilizó para la definición de la muestra el sistema semana compuesta no probabilística, en el cual mediante un procedimiento sistemático logra revisar el todo. Para su desarrollo, "se selecciona un determinado día de la primera semana (por ejemplo lunes), entonces de la segunda semana se selecciona el día subsecuente (el martes), de la tercera semana el

\footnotetext{
29. Id.

30. Giovanni Bohórquez-Pereira. El tema ambiental en la agenda informativa: análisis de contenido de la prensa local año 2005. Puente 2. 2009. Pág. 63.
} 
miércoles y así sucesivamente...." ${ }^{31}$ De esta manera, se logró que los periódicos seleccionados representaran el universo del objeto de estudio y se obtuvo una muestra con criterio de validez.

La selección de los periódicos a estudiar se inició un domingo y finalizó un miércoles. Cada mes fue representado por una semana de lunes a domingo y el ejemplar asignado se revisó en busca de las unidades de análisis desde la primera hasta la última página, se eximió la sección de clasificados. Durante el 2011, circularon 519 ejemplares, de los cuales 363 fueron de Vanguardia Liberal y 156 de El Frente, tal como se observa en la tabla 1. La diferencia numérica entre uno y otro se centra en las ediciones. Mientras el primero circula 363 de los 365 días del año (no hay publicación los días festivos 1 de enero y Jueves Santo), El Frente circula tres veces por semana, durante las 52 semanas del año. En total, la muestra fue de 52 ejemplares promedio, para un total de 104, lo cual representa el 20\% del total de periódicos divulgados ese año.

Tabla 1. Periódicos que circularon y fueron analizados

\begin{tabular}{|l|c|c|}
\hline \multicolumn{1}{|c|}{ Periódico } & $\begin{array}{c}\text { Número de periódi- } \\
\text { cos que circularon }\end{array}$ & $\begin{array}{c}\text { Número de periódi- } \\
\text { cos analizados }\end{array}$ \\
\hline Vanguardia L. & 363 & 52 \\
\hline El Frente & 156 & 52 \\
\hline Total & 519 & 104 \\
\hline
\end{tabular}

Fuente: elaboración propia

\section{Participantes}

El estudio, por ser de carácter hermenéutico y utilizar la técnica de análisis de contenido, lleva a asumir como participantes a los periódicos que por fecha $-\mathrm{y}$ de acuerdo con el método de semana compuesta- fueron seleccionados para estudiar.

Se aprecia entonces que el proceso cumple con exhaustividad, representatividad, homogeneidad y pertinencia, los cuales en palabras de Cabero y Loscertales, ${ }^{32}$ son principios fundamentales al momento de realizar estudios de análisis de contenido.

31. Francisco de Jesús Aceves. Problemas metodológicos en el estudio de la cobertura informativa de los medios en los procesos electorales. Hacia una validación de la muestra. B. Russi. ANUARIO DE INVESTIGACIÓN DE LA COMUnicación. (2003). Pág. 263.

32. Julio Cabero y Felicidad Loscertales, Dirs. Elaboración de un SISTEMA CATEGORIAL DE ANÁLISIS DE CONTENIDO PARA ANALIZAR LA IMAGEN DEL PROFESOR Y LA ENSEÑANZA DE LA PRENSA. Universidad de Sevilla y Huelva. (1995). Disponible en http://edutec.rediris.es/documentos/1996/ANALISIS.htm
El primer principio (exhaustividad) se logró en el procedimiento de indagar por diversos medios para localizar y acceder a los periódicos seleccionados. La representatividad se alcanzó, ya que todos los meses del 2011 hicieron parte del estudio. De igual manera, la búsqueda de información relacionada con el tratamiento periodístico dado por los periódicos a los niños y niñas víctimas del conflicto en todas las páginas y secciones del diario, llevó a ubicar un total de 56 notas que representan los diferentes géneros periodísticos.

Se halló la homogeneidad cuando la unidad a estudiar cumplió con las características de estructura y contenido que se exigen en la información periodística relacionada con menores víctimas del conflicto. Por último, fue pertinente dado que los textos y sus componentes hicieron que los objetivos específicos se alcanzaran y con ellos el objetivo general.

\section{REsultados}

El estudio se llevó a cabo con 56 notas periodísticas, 75\% del diario Vanguardia Liberal y 25\% de El Frente. La información analizada involucra a toda la diversidad de actores participantes de la guerra en Colombia tales como Estado, subversión, paramilitares, bandas criminales (Bacrim), grupos de narcotraficantes y delincuencia común, quienes involucran en sus acciones a niños y niñas.

Los periódicos presentan su información en páginas y en cada una de ellas desarrollan aspectos particulares, a los cuales denominan sección. En los periódicos analizados, se encontró que la sección Judicial fue donde se registró el mayor porcentaje de información relacionada con niños, niñas y conflicto armado (38\%). Le siguió la sección Nacional con 27\%. La sección Metropolitana obtuvo 7\%, mientras que las secciones Piedecuesta y Floridablanca registraron 4\%. Un 3\% las secciones Regional e Informe, y las secciones Barrancabermeja y Política un 1\%. Sin registro estuvieron las secciones Local, Editorial, Entretenimiento, Deportes y Opinión (ver gráfica 1). 


\section{Sección del periódico}

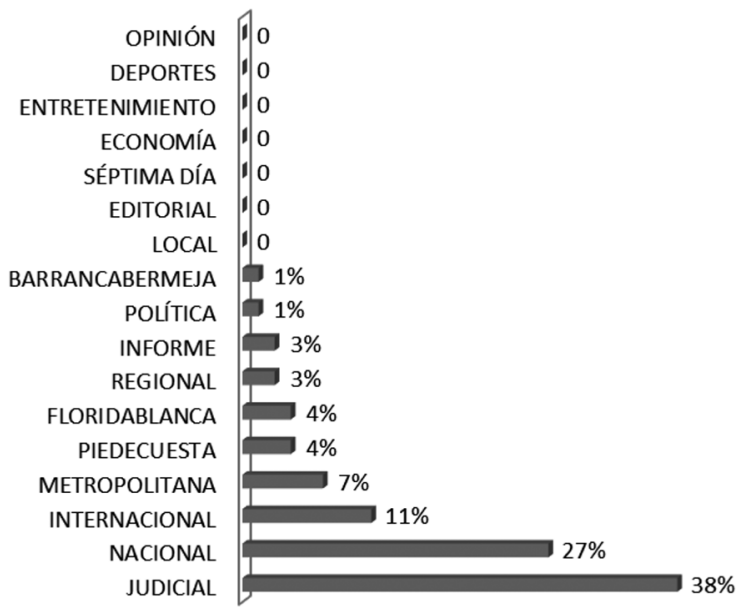

Gráfica 1. Sección de mayor registro Fuente: elaboración propia

En dichas secciones, el género periodístico más utilizado fue la noticia con un 58\%, seguido de la noticia breve o cápsula con un $25 \%$, y el reportaje con una incidencia del 6\%. Con una incidencia mínima aparece la crónica con un 1\%, y los géneros de entrevista, perfil, columna o editorial no se encuentran evidenciados (ver gráfica 2).

\section{Géneros periodísticos}

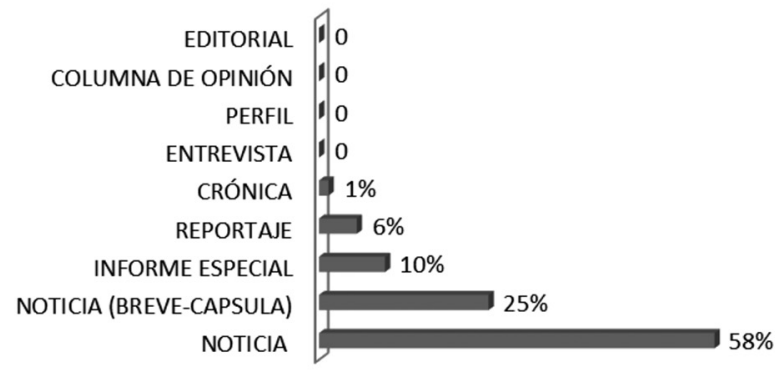

Gráfica 2. Géneros periodísticos registrados Fuente: elaboración propia

Con respecto a la extensión del texto, se destacó el rango 5-15 cm, con el 74\%. El 19\% de las notas informativas se inscribió en el rango 16 a $25 \mathrm{~cm}$ y un $7 \%$ tuvo una extensión entre 26 a $35 \mathrm{~cm}$. No se registró información en los rangos $36-45 \mathrm{~cm}$, ni de más de $46 \mathrm{~cm}$.
Ahora bien, se hizo necesario consultar y definir la victimización infantil y sus diversas tipologías, a fin de analizar algunos rasgos particulares expuestos en las notas periodísticas seleccionadas. Para ello, se acudió a Pereda, Abad y Guilera ${ }^{33}$ y a sus tipologías: exposición a violencia comunitaria, y subcategorías: menores desplazados y refugiados y menores soldados o utilización de los niños y las niñas en conflictos armados y atentados terroristas. Otras dos categorías fueron maltrato o explotación laboral y corrupción como forma de victimización infantil.

A partir de lo anterior, los resultados indicaron que la categoría de mayor registro para el caso particular de niños fue Maltrato o explotación laboral con 28\%, Menores desplazados y refugiados, Menores soldados o utilización de los niños y las niñas en conflictos armados $y$ atentados terroristas y Corrupción como forma de victimización infantil, las cuales se registraron en un $24 \%$.

En niñas, la tipología de mayor registro fue $\mathrm{Me}$ nores desplazados y refugiados con $37 \%$, seguida de Maltrato o explotación laboral con 31\%. Las tipologías Menores soldados o utilización de los niños y las niñas en conflictos armados y atentados terroristas y Corrupción como forma de victimización infantil, registraron $16 \%$ cada una.

Los niños y niñas son referenciados en la información publicada como sujeto víctima del conflicto armado de seis maneras. Para el caso de las niñas son registradas en $50 \%$ como víctimas en la subcategoría esclava doméstica y sexual. En un 32\%, se referencian como víctimas de desaparición forzada, y en la subcategoría víctima secuestrada en un $18 \%$ de los casos. No se registran las niñas víctimas en las subcategorías huérfanas, mutiladas o desplazadas.

En el caso de los niños el porcentaje mayoritario sigue siendo la subcategoría víctimas como esclavo doméstico y sexual con un $47 \%$, la subcategoría víctima desaparición forzada registró en un $22 \%$, y víctima secuestro, un 13\%. Además, se registran las subcategorías víctimas niños mutilados y desplazados en un $9 \%$ cada una. No hubo registro de la subcategoría víctimas niños huérfanos por el conflicto (ver gráfica 3).

33. Noemí Pereda, Judit Abad y Georgina Guilera. Victimología DeL DESARROLLO. INCIDENCIA Y REPERCUSIONES DE LA VICTIMIZACIÓN Y LA POLIVICTIMIZACIÓN DE LOS JóvENES CATALANES. Generalitat de Cataluña. Departamento de Justicia. Centro de Estudios Jurídicos y Formación Especializada. (2012). Págs. 16-25. 


\section{Sujeto- víctima}

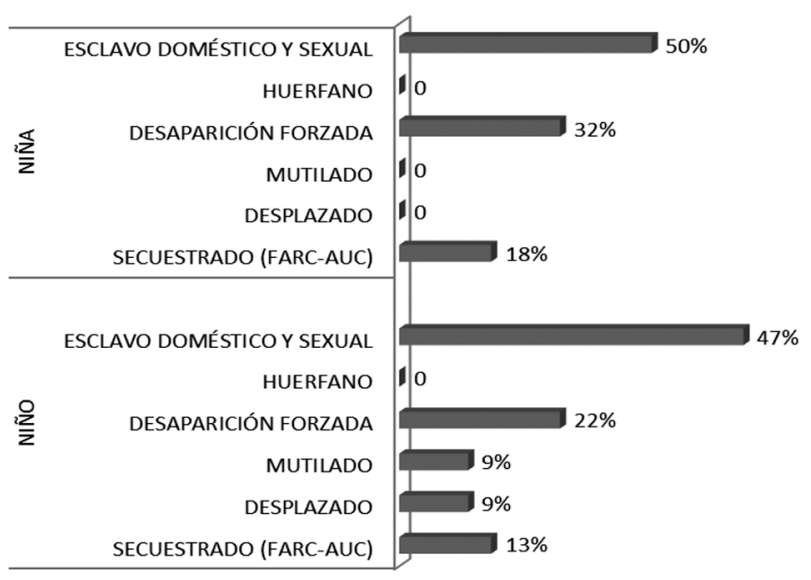

Gráfica 3. Datos que se publicaron sobre el sujeto Fuente: elaboración propia

A partir de las subcategorías esclavo doméstico y sexual, capturado, informantes, desvinculado, desmovilizado y combatiente, se revisó la información para ubicar las referencias que del menor se hace en la información como victimario.

En el caso de las niñas, la única subcategoría victimario registrada es capturada, mientras que en los niños dicha subcategoría arrojó el $81 \%$. Las subcategoría desvinculados de grupos armados registró un $14 \%$ y como desmovilizados un 5\%. No se registran en niños, ni en niñas, las subcategorías victimarios informantes, esclavos domésticos y sexuales o combatientes.

En lo relativo a la familia del niño y la niña, un 33\% de la información difundió datos, de los cuales sobresalió el registro del nombre de padre-madre-hermanos (13\%), la ubicación geográfica de residencia (11\%), edades de los familiares (7\%) y la ocupación-labor (2\%).

\section{Discusión}

Las categorías discursivas identificadas en los dos medios estudiados dan cuenta de la forma como estos comprenden la situación de los niños y niñas afectados por el conflicto armado, así como el mensaje que está dejando en la sociedad en general con sus enunciados.

A partir del análisis de la información, se pone de manifiesto que los medios estudiados abordan el tema de manera tangencial, reportando a la opinión pública los hechos que hacen noticia indistintamente, sin ofrecer un énfasis particular en el tema de la niñez.
En esta medida, si la intención de los medios fuese exponer ante la sociedad la situación de los niños afectados por el conflicto, es bastante probable que no alcance ese objetivo, dado que la tendencia de la información, de acuerdo con el ítem analizado, es de informar sobre hechos que ocurren en el marco de las circunstancias del país, pero no en procura de una reflexión que conduzca a acciones en pro de la defensa y garantía de los derechos de los niños y niñas.

En este sentido, vale la pena destacar que el género noticia fue el más utilizado con $58 \%$, le sigue la brevecápsula con $25 \%$, informe especial registró un $10 \%$, un $6 \%$ el reportaje y $1 \%$ la crónica. Los géneros entrevista y perfil, así como los denominados de opinión (editorial y columna), no registran presencia en la muestra estudiada. Los datos expuestos permiten evidenciar que la difusión del tema no alcanza a tener los mismos espacios de interés que suscitan otros hechos del orden local y nacional.

En las limitadas líneas y centímetros (5 a $15 \mathrm{~cm}$ ), y del reducido número de notas informativas publicadas por los periódicos locales estudiados (56), se logró evidenciar que los niños y niñas son presentados a los lectores más en función de víctimas y victimarios de una guerra, que de sujetos integrantes de una sociedad. Si la pretensión o intención no manifiesta de los medios impresos locales es exponer a la sociedad situaciones sociales complejas como lo son las violencias y, en particular, el conflicto armado colombiano en busca de reflexión, se puede afirmar que se quedan cortos al utilizar para ello 5 a $15 \mathrm{~cm}$ de texto (74\%). Tanto niñas (37\%), como niños (24\%) se presentaron en los medios impresos - desde los parámetros de Pereda, Abad y Guilera - como perseguidos y desplazados de su comunidad de origen por causas de violencia política, sufriendo importantes situaciones de crueldad, racismo y prejuicios culturales. ${ }^{34}$

De acuerdo con este panorama, se observó cómo la sociedad que lee periódicos con regularidad no advierte en los textos una diferencia entre víctimas y victimarios, y se refuerza cuando la sección Judicial con un 38\% detalla los hechos en los que están involucrados de manera directa o indirecta los niños colombianos.

Titulares tales como "Escolares extorsionistas", ${ }^{35}$ "Menores autores de masacre", 36 "Frustraron el secuestro

\footnotetext{
34. Id.

35. Escolares extorsionistas. Vanguardia Liberal. Septiembre 29 de 2011.

36. Menores autores de masacre. El Frente. Enero 19 de 2011.
} 
de una joven de 17 años y de sus pequeños hijos"37 son ejemplos que llevan a reflexionar sobre la manera en que la sociedad en general está mirando el conflicto y qué tanto absuelve o condena la presencia de los menores en él.

Sin duda alguna, en el conflicto armado, los miembros de la familia más afectados son los niños, pues el traslado a un entorno extraño y hostil hace que estas pequeñas víctimas dejen morir, más inconsciente que conscientemente, los sueños que habían comenzado a forjar. No son extraños los casos de suicidio de adolescentes, el aumento de embarazos de niñas que apenas comienzan a vivir, la vinculación a grupos armados, alcoholismo y prostitución, entre otros. Los medios de comunicación, y en especial la prensa escrita, no pueden seguir tratando estos problemas como elementos que atraen la atención del público y que sirven de "gancho" periodístico sin respetar la dignidad humana de los menores que, en realidad, son las víctimas de ese inaceptable y aberrante fenómeno social del desplazamiento.

Más que discutir la estructura y algunos componentes de redacción de la información, lo lamentable es evidenciar a través de lo publicado que en Colombia no se protegen ni respetan los derechos de los niños y las niñas. Es evidente también que el respeto y la protección a esta población por parte de padres y cuidadores, tanto en sectores urbanos como rurales del país, es reducida y que en algunos casos son los propios progenitores o familiares cercanos quienes los inducen a participar en hechos delictivos, o en su defecto a abusar de ellos o explotarlos. Lamentable.

La Ley 12 de 1991 que adopta la Convención sobre los Derechos del Niño, respecto al comportamiento de los medios de comunicación, determina en su art. 17:

Los Estados Partes reconocen la importante función que desempeñan los medios de comunicación y velarán por que el niño tenga acceso a información y material procedentes de diversas fuentes nacionales e internacionales, en especial la información y el material que tengan por finalidad promover su bienestar social, espiritual y moral y su salud física y mental. Con tal objeto, los Estados Partes:

a) Alentarán a los medios de comunicación a difundir información y materiales de interés social y cultural para el niño, de conformidad con el espíritu del artículo 29;

37. Frustraron el secuestro de una joven de 17 años y de sus pequeños hijos. VANGUardia Liberal. Mayo 5 de 2011. b) Promoverán la cooperación internacional en la producción, el intercambio y la difusión de esa información y esos materiales procedentes de diversas fuentes culturales, nacionales e internacionales;

c) Adelantarán la producción y difusión de libros para niños;

d) Adelantarán a los medios de comunicación a que tengan particularmente en cuenta las necesidades lingüísticas del niño perteneciente a un grupo minoritario o que sea indígena;

e) Promoverán la elaboración de directrices apropiadas para proteger al niño contra toda información y material perjudicial para su bienestar, teniendo en cuenta las disposiciones de los artículos 13 y 18.

A lo que se suma el incumplimiento por parte de algunos medios de las normas nacionales e internacionales sobre la protección de la integridad del menor. Los periodistas, que son los responsables y deciden qué y dónde publicar, deben asumir el cumplimiento de la Ley 1098 del $2006^{38}$ y realizar, como lo señala Álvaro Sierra, un cubrimiento periodístico respetuoso, no sensacionalista, contextualizado y desde una perspectiva de derechos, sin que sea lastimera:

En una de las peores naciones del mundo en materia de niñez y conflicto armado como Colombia, que los periodistas y los medios asuman a los niños soldados como personas y los traten con el respeto y el cuidado que merecen como víctimas del conflicto armado es una obligación moral y profesional de lo más elemental. ${ }^{39}$

Otro aspecto significativo para resaltar en esta construcción discursiva es la no presencia de los géneros de opinión. Desde una perspectiva imparcial, llama la atención que los denominados líderes de opinión en los periódicos estudiados no realicen pronunciamientos sobre el conflicto armado, y en particular sobre los niños y niñas. En la muestra estudiada se evidencian discusiones y llamados de atención por parte de los columnistas y editorialistas sobre temáticas relacionadas con corrupción, gestión de gobernantes y propuestas para cambios sociales de la ciudad y la región. Sin embargo, se olvida el fenómeno social del reclutamiento a menores, intento de secuestro, maltrato infantil en sus diversas manifestaciones, desmovilización de los

\footnotetext{
38. Ley 1098 de 2006, supra, nota 2.

39. Álvaro Sierra. Elementos del contexto legal. Niños vinculados AL CONFLICTO: CUBRIMIENTO PERIODístico RESPONSABLE. Corporación Medios para la Paz y Fundación Colombia Multicolor. (2009). Pág. 34.
} 
menores, y los efectos que estos generan en los infantes, sus familias y la sociedad en general.

Se ha manifestado desde la teoría que el líder de opinión es una figura de respeto y orientadora de las sociedades. En el caso puntual del periodismo, autores como Kimball Young, citado por Raúl Rivadeneira, reseña que uno de los rasgos que caracterizan a los líderes y sus interacciones con las masas es que "pueden ser los primeros en plantear o definir una cuestión".40

A su vez, Cándido Monzón, citado por Iván Abreu, al listar las diez características que se le atribuyen a un líder de opinión destaca la relación entre los grupos sociales y los medios de comunicación, y señala que "los líderes de opinión actúan como correa de transmisión entre la comunicación social y la comunicación de grupo, y la comunicación va desde los medios hasta los líderes de opinión y de estos al resto de grupo" ${ }^{41}$.

Sin embargo, es también necesario recordar la advertencia hecha en la década de los setenta por Joseph Klapper, en sus estudios respecto a la incidencia del líder de opinión, pues por una parte puede actuar como refuerzo y protección a las normas sociales y del statu quo, como también apoya y dinamiza posibles cambios en el orden social: "Como vía de acceso o transmisor de los contenidos de las comunicaciones de masas, el líder de la opinión puede transmitir o aprobar el material acorde con las normas de su grupo y dejar de transmitir $\mathrm{o}$ atacar el material opuesto a ellas" ${ }^{42}$

Surge, entonces, la discusión y la pregunta del comportamiento de los columnistas y de los editoriales revisados en la muestra estudiada, quienes no escribieron ni reflexionaron sobre el tema de estudio. Desde una perspectiva emotiva, se podría afirmar que la temática del conflicto armado en Colombia, y en particular la presencia de menores en él carece de importancia, de manera que avalan y refuerzan la existencia de este. Desde otra mirada, se puede decir que no es contundente señalar el desinterés por parte de los líderes de opinión frente al tema, sino que la aleatoriedad de la muestra llevó a presentarse esta situación.

No obstante, llama la atención que al comparar trabajos del mismo tipo, la situación es cercana o similar. Guardadas las proporciones, lo encontrado en el pre-

40. Véase Rivadeneira, supra, nota 17. Pág. 197.

41. Iván Abreu Sojo. EL ESTUdiO DE LA OPINIÓN PÚBLICA: ESPACIO PÚBLICO Y Medios DE COMUNiCACión. 3a ed. Vadell Hermanos Editores. (2009). Pág. 101.

42. Joseph Klapper. Efectos de las comunicaciones de MaSAS. Aguilar. (1979). Pág. 35. sente estudio se asemeja a lo encontrado en el año 2010 por la Agencia PANDI. Dicha organización realizó un estudio a 11 medios impresos en Colombia centrado en el registro informativo que los medios hacen en relación con la infancia.

Los investigadores encontraron que de las 9593 notas informativas recolectadas, solo $3,4 \%$ tuvieron relación con la niñez en el conflicto armado. De dicho porcentaje, la noticia (con el $87,5 \%$ ) fue el género periodístico de mayor presentación, seguido del artículo de opinión con 9,5\%, la entrevista con $1,5 \%$ y el editorial con $1,5 \%$. Frente a este último porcentaje enfatizan: "Se trata de un dato que no debe pasarse por alto ya que mucha de la atención de los editorialistas está puesta en el conflicto armado colombiano, pero los niños y niñas parecen invisibles". ${ }^{43}$

El orden sistemático y la rigidez propia de la técnica hicieron que el análisis de contenido permitiera desarrollar prácticas investigativas como la efectuada y a la vez alcanzar lecturas de la sociedad actual desde el texto publicado.

\section{Conclusiones}

A partir del análisis de contenido de tipo semántico, se logró entender cómo los niños y niñas víctimas del conflicto armado en Colombia fueron ubicados en las páginas de los periódicos locales, así como responder a las preguntas ¿con qué frecuencia se hizo referencia a ellos?, ¿en qué tipo de hechos se involucraron y qué características de los niños y niñas víctimas del conflicto se registraron durante el lapso analizado?

Se evidencia que el ejercicio periodístico está siendo reducido a la automatización o a la lógica de la producción de información, es decir, se advierte un ciclo mecánico en el que día a día se muestra una situación similar (los niños, niñas y adolescentes en medio de conflicto armado y social), y lo único que cambia son los nombres o lugares donde se desarrolla el hecho. No se responden preguntas más allá de quién, del cómo, del cuándo, del dónde, del qué, se niega el por qué y hasta el para qué.

Se requiere entonces revisar, en compañía de los periodistas, los criterios que definen las agendas informativas, indagar sobre las causas que llevan a la limitación de registro en extensión de texto y presentación

43. Norato y Farfán, supra, nota 15. Pág. 41 
de la información (género periodístico), al igual que el seguimiento de los hechos.

Es importante estudiar y preguntarle al otro. Las audiencias, en este caso, los lectores, deben ser consultados. ¿Qué análisis realizan de las informaciones que presentan los periódicos de los niños y niñas en el conflicto armado? y iconsideran que lo informado es acertado? Estos y otros interrogantes se deben hacer, y tanto la academia como los medios masivos de información, deberían promover respuestas desde lo científico, es decir, realizar investigaciones de carácter cualitativo que permitan escuchar a quien debemos nuestra labor diaria: la gente.

Quedan en discusión elementos propios del periodismo que merecen reflexión. Estos son algunos que se proponen como futuras investigaciones:

- ¿Qué ocurre en el mundo de hoy con la imparcialidad informativa?, ¿es real o se convirtió en compromiso desde lo individual?

- ¿Qué aportes dan los medios a la discusión social de problemas como el aquí estudiado? ¿Se deben quedar en el registro noticioso o deben trascender mediante la discusión pública?

- La interlocución con los lectores y públicos es hoy una obligación a asumir por parte de los medios de información. Negarlo o no hacer algo al respecto es una posibilidad de aislamiento y de reducción de lectores y suscriptores.

- La globalidad permite en nuestros tiempos, además de acceder a las cosas, conocer de cerca y con otras fuentes los hechos que ocurren en nuestro entorno y en nuestra región sudamericana. ¿Cuentan los comunicadores-periodistas actuales y futuros con las competencias necesarias para asimilar, comprender, jerarquizar y proponer contenidos que logren informar de mejor manera a la sociedad?

Las escuelas y facultades de comunicación social y periodismo deben formularse en sus programas la pregunta de cómo mantener y fortalecer los ámbitos de la investigación formativa, tanto en la aplicación de metodologías, como en el análisis del contenido y discurso, así como formular y ofertar a egresados y estudiantes en formación cursos, seminarios, especializaciones en temas relacionados con la normatividad jurídica, no sólo en lo relacionado con los niños, niñas y adolescentes, sino también con temas tales como conflicto y posconflicto, familia y derechos sociales.
El presente estudio realiza una primera iniciativa al crear un blog ${ }^{44}$ de tipo científico, el cual, además de hacer públicos los resultados de la investigación, se proyecta como fuente de consulta en aspectos como normatividad. Cuenta con materiales de apoyo (videos y boletines de prensa), producidos por organismos nacionales e internacionales que trabajan en la defensa de los derechos de los niños.

Finalmente, es pertinente hacer una invitación a los periodistas y comunicadores a consultar fuentes, $y$ en particular las denominadas de autoridad ${ }^{45}$ quienes trabajan diariamente en conocer e interpretar las dinámicas sociales locales, y mundiales. La región y el país cuentan con centros, institutos y grupos de investigación de tradición y excelencia en estos campos.

\section{REFERENCIAS}

Albert Chillón. El giro lingüístico en periodismo y su incidencia en la comunicación periodística. CUADERNOS DE LA INFORMACIÓN 14. 2001.

Álvaro Sierra. Elementos del contexto legal. Niños vincuLADOS AL CONFLICTO: CUBRIMIENTO PERIODÍSTICO Responsable. Corporación Medios para la Paz y Fundación Colombia Multicolor. (2009).

Carlos Sandoval. Investigación cualitativa. Programa DE Especialización en Teoría, Métodos y Técnicas de InVEstigación Social. Arfo Editores. (2002).

Coalición contra la vinculación de niños, niñas y jóvenes al conflicto armado en Colombia. Boletin estadístico 20092011. Boletín de Monitoreo Niñez y Conflicto Armado 9. Noviembre de 2012. Disponible en: http:// www.coalico.org/Bonca_coalico_9.pdf

Corporación para el Desarrollo del Oriente. Desarme, desmovilización y reintegración en Santander. Boletín 1. 2012. Disponible en http://www.corporacioncompromiso.org/index.shtml?apc $=$ e1-1--- \&x=599

Corte Constitucional de Colombia. Sentencia T-453/13. (M. P. Nilson Pinilla Pinilla; julio 15 de 2013). Disponible en http://www. corteconstitucional.gov.co/ relatoria/2013/T-453-13.htm

Escolares extorsionistas. VANGUARDiA Liberal. Septiembre 29 de 2011.

44. Véase http://ninosdelconflicto.blogspot.com

45. Stella Martini. Periodismo, noticia y noticiabilidad. Enciclopedia LATINOAMERICANA DE SOCIOCULTURA Y COMUNICACIÓN. Grupo Editorial Norma. (2000). Pág. 66. 
Francisco de Jesús Aceves. Problemas metodológicos en el estudio de la cobertura informativa de los medios en los procesos electorales. Hacia una validación de la muestra. B. Russi. Anuario de investigación de la COMUniCACIÓN. (2003).

Frustraron el secuestro de una joven de 17 años y de sus pequeños hijos. Vanguardia Liberal. Mayo 5 de 2011.

Giovanni Bohórquez-Pereira. El tema ambiental en la agenda informativa: análisis de contenido de la prensa local año 2005. Puente 2. 2009.

Guillermo López. COMUniCACión Electoral y FORMACIÓN DE OPINIÓN PÚBLICA: LAS ELECCIONES GENERALES DE 2000 EN LA PRENSA ESPAÑOLA. Universidad de Valencia. (2004).

Iván Abreu Sojo. El ESTUdio DE LA OPINIÓN PÚBLICA: ESPACIO PÚBLICO Y MEdios DE COMUNiCACIÓN. 3a ed. Vadell Hermanos Editores. (2009).

Joseph Klapper. Efectos de LAS COMUniCACIONES DE MAsas. Aguilar. (1979).

Juan Carlos Amador-Baquiro. El intersticio de la víctimavictimario: un análisis de los procesos de subjetivación de cuatro desvinculados de grupos armados en Colombia. Universitas humanística 69. Junio de 2010.

Julián Aguirre y Miguel Álvarez-Correa, Eds. Guerreros SIN SOMBRA: NIÑOS, NIÑAS Y JÓVENES VINCULADOS AL CONFLICTO ARMADO. Instituto Colombiano de Bienestar Familiar y Procuraduría General de la Nación. (2002).

Julio Cabero y Felicidad Loscertales, Dirs. ElaboraCión DE UN SISTEMA CATEGORIAL DE ANÁLISIS DE CONTENIDO PARA ANALIZAR LA IMAGEN DEL PROFESOR Y LA ENSEÑANZA DE LA PRENSA. Universidad de Sevilla y Huelva. (1995). Disponible en http://edutec.rediris.es/documentos/1996/ANALISIS.htm

Klaus Krippendorff. Metodología DE ANÁLISIS DE CONTENIDO, TEORÍA Y PRÁCTICA. (1997).

Ley 12 de 1991. Por la cual se adopta la Convención sobre los Derechos del Niño. Enero 22 de 1991. Do N. 39640.

Ley 1098 de 2006. Por la cual se expide el Código de la Infancia y la Adolescencia. Noviembre 8 de 2006. Do N. ${ }^{\circ}$ 46446. Disponible en http://www.alcaldiabogota.gov. co/sisjur/normas/Norma1.jsp?i=22106

Mauro Wolf. LA INVESTIGACiÓN DE LA COMUNiCACióN DE MASAS: CRÍtICA Y PERSPECTIVAS. Editorial Paidós. (1987).

Mary Correa. El lenguaje de los medios que intensifica el conflicto armado colombiano. Reflexión PolíticA 19. Junio de 2008. Págs. 106-113.
Maxwell McCombs y Donald Shaw. The Agenda-Setting Function of Mass Media. Public Opinion Quarterly 2. Verano de 1972. Págs. 176-187.

Medios para la Paz. LAs trampas de LA GUerRa: PERIODismo y CONFLicto. Corporación Medios para la Paz. (2001).

Menores autores de masacre. El Frente. Enero 19 de 2011.

Noemí Pereda, Judit Abad y Georgina Guilera. ViCtimoloGÍA DEL DESARROLLO. INCIDENCIA Y REPERCUSIONES DE LA VICTIMIZACIÓN Y LA POLIVICTIMIZACIÓN DE LOS Jóvenes Catalanes. Generalitat de Cataluña. Departamento de Justicia. Centro de Estudios Jurídicos y Formación Especializada. (2011).

Orlando D’Adamo, Virginia García y Flavia Freidenberg. Medios de COMUNiCACIÓN Y OPINIÓN PÚBliCA. McGraw-Hill. (2007).

Óscar Misle. LA NIÑEZ y LA ADOLESCENCIA EN LOS MEDIOS: ¿CUÁNDo sí Y CUÁNdo NO? Ediciones El Papagayo. (2008).

Raúl Rivadeneira. La OPINIÓN PÚBLICA. ANÁLISIS, ESTRUCTURA Y MÉTODOS PARA SU ESTUdIO. Editorial Trillas. (2007).

Roberto Hernández Sampieri, Carlos Fernández y Pilar Baptista. Metodología de la investigación. McGraw Hill. (2010).

Stella Martini. Periodismo, noticia y noticiabilidad. ENCICLOPEDIA LATINOAMERICANA DE SOCIOCULTURA Y COMUNICACIÓn. Grupo Editorial Norma. (2000).

United Nations International Children's Emergency Fund. La Convención sobre los Derechos del Niño (CDN). (2013). Disponible en http://www.unicef.org/ lac/CDN.pdf

Walter Lippmann. LA OPINIóN PÚBLICA. Editorial C. de Langre. (2003).

Ximena Norato y Alexandra Farfán, Eds. LA HUELla DE LA niñeZ En la prensa COlombiana 2008. Agencia de Comunicaciones Periodismo Aliado de la Niñez, el Desarrollo Social y la Investigación (PANDI), Fundación Nuevo Periodismo Iberoamericano, Plan y Unicef. (2008).

Ximena Norato y Alexandra Farfán, Eds. LA HUELla DE LA niñeZ En la prensa COlombiana 2010. Agencia de Comunicaciones Periodismo Aliado de la Niñez, el Desarrollo Social y la Investigación (PANDI), Personería de Medellín y Unicef. (2010). 